\title{
CareTrack: an Application-Based Method of Documentation for Improving Communication in Cancer Care
}

\author{
Vineet $\mathbf{S}^{1}$, Khan $\mathrm{F}^{1}$, Mirza $\mathrm{I}^{1}$, Black $\mathbf{M}^{2}$, Raphael $\mathrm{J}^{2}$, Vincent $\mathbf{M}^{2}$, Blanchette $\mathrm{P}^{2}$, Kuruvilla $\mathbf{S}^{2}$, Sanatani $\mathbf{M}^{2}$ and Younus $\mathrm{J}^{2, *}$ \\ ${ }^{1}$ Western University, London, Ontario, Canada \\ ${ }^{2}$ London Regional Cancer Program, London Health Sciences Centre, London, Ontario, Canada
}

"Corresponding author: Jawaid Younus, Medical Oncologist, London Regional Cancer Program, London Health Sciences Centre 800 Commissioners Road E, London, Ontario, N6A5W9, Canada, Phone: (519) 685-8500 x53327; E-mail: Jawaid.Younus@lhsc.on.ca

Received: 11 Mar, 2020 | Accepted: 15 Apr, 2020 | Published: 20 Apr, 2020

Citation: Vineet S, Khan F, Mirza I, Black M, Raphael J, et al. (2020) CareTrack: an Application-Based Method of Documentation for Improving Communication in Cancer Care. Int J Cancer Res Mol Mech 5(1): dx.doi.org/10.16966/2381-3318.146

Copyright: (C) 2020 Vineet S, et al. This is an open-access article distributed under the terms of the Creative Commons Attribution License, which permits unrestricted use, distribution, and reproduction in any medium, provided the original author and source are credited.

\section{Abstract}

A patient being able to accurately understand and recall medical information correctly has been shown to improve outcomes. However, many studies suggest that patient understanding about the disease and its management in oncology tends to be poor. Our study aimed to implement CareTrack, a simple, yet complete app-based information package that provides an initial appointment summary for patients with lung cancer. An iPad with the CareTrack application was provided to medical oncologists to complete at the end of the initial consultation. A printout copy was provided to patients to take home. One-week later a patient satisfaction questionnaire was administered over the phone. Five oncologists and 37 patients participated in the study. Our primary objective was to assess feasibility of the CareTrack application from the physician perspective. The average physician time to complete the CareTrack application form for each patient was 1 minute and 23 seconds, thus demonstrating that this is a quick and easy tool for physicians. Our secondary objective was to assess patient satisfaction. $97 \%$ of patients found the information provided to be useful and $100 \%$ of patients found it easy to understand. Most patients were found to refer to the CareTrack application form more than once (33/36) and used the form to remember information (34/37). Importantly, $95 \%$ of patients were comfortable seeing their cancer information displayed on the application and $86 \%$ of patients would like to receive additional information in the future if their cancer management changed. CareTrack was found to be user friendly and able to effectively provide lung cancer patients with a comprehensive yet easy-to-understand summary of their initial consultation.

Keywords: Lung cancer; Initial oncology consultation; Improving patient education; Improving patient satisfaction; Application-based technology; Pilot study

\section{Introduction}

It is well understood that cancer patients want as much information as possible regarding their disease [1,2]. Patients who wanted to be involved in treatment discussions and had the desire for a maximum amount of information had the best overall treatment outcomes [1]. Unfortunately, many studies have shown as much as $40-80 \%$ of the information relayed by healthcare professionals is forgotten [3]; patients will have similar needs in terms of information throughout the treatment, due to the fact that they have forgotten what was mentioned at the first visit after their radiation therapy [2]. Given that patients are not able to accurately recall information about their treatment; this leads to a feeling of a lack of information for patients. This feeling of being 'left in the dark' has been found to lead to increased anxiety and distress [4]. Being unable to accurately understand and recall medical information correctly has been shown to lead to negative patient stratification and can influence the patient's treatment choices [4]. Overcoming this information barrier leads to better compliance, decreased anxiety [4] and improved patient satisfaction [2].

More recently, mobile tablet and smartphone applications (i.e., mobile health) offer a unique opportunity to improve on the quality and safety of healthcare delivered by healthcare providers to patients $[5,6]$. Many mobile health technologies have been developed in an effort to help bridge this information gap for patients in healthcare. A study at the Abramson Cancer Center at the University of Pennsylvania in 2018 found that $74 \%$ of cancer patients reported use of mobile devices and $39 \%$ of patients expressed an interest in receiving additional supportive care information via smartphone/ tablet applications [7]. To date, mobile health has been utilized in the prevention, diagnosis, decision, treatment and follow-up phases of the patient healthcare journey and has the potential to improve patient quality of life, deliver more appropriate care and to decrease 
the burden on the healthcare system by assisting clinical processes [8]. A 2018 review concluded that mobile health has promising potential as a tool for cancer patients and survivors to help overcome current barriers such as difficulty communicating with providers and inadequate communication between patients and providers regarding symptoms [9]. However, despite providing healthcare providers a unique opportunity to deliver high-quality care, mobile health presents some concerns such as organizational barriers in the practice, regulatory oversight, privacy, confidentiality, and ownership of data, and integration into existing health record platforms [5]. Such concerns need to be addressed with mobile health applications in order to be safe and effective in the clinical setting to align with both healthcare provider and patients needs.

Although many medical/oncology-related applications are currently available, these applications tend to be limited to certain phases and technologies of the healthcare journey [8] with many being designed for patient use in order to manage their health information including tracking appointments, medications and symptoms. A review of relevant literature examining the role of mobile health in cancer supportive care concluded that it is underutilized in oncology despite its potential to assist patients [8]. Currently, there are no other competitor applications on the market that are designed to be completed by the physician/healthcare team to give the patient a summary of their appointment, disease and detailed information of their treatment plan. Thus, it is vital for healthcare providers to utilize interventions that are effective in improving patient information reception and understanding.

To address this unmet need in the oncological field, we have developed an iPad-based application entitled 'CareTrack'. The CareTrack application has an easy-to-use interface for healthcare providers, maintains confidentiality and produces an email and/or printout summary of the appointment with information on diagnosis, stage and treatment for patients to take home. Our pilot study aims to implement the use of the CareTrack application and evaluate its efficacy in terms of feasibility, as well as patient and physician satisfaction.

\section{Objectives}

The main objective of this study was to assess the feasibility of the CareTrack application, and to determine if physicians could reasonably take the time to fill out the CareTrack application in their busy clinics. As secondary objectives, we aimed to assess satisfaction from both patients as well as physicians. For patient satisfaction, we aimed to assess whether the CareTrack application was actually useful to patients, by assessing their satisfaction with the application and appraising how well they understood the information presented to them. For physicians, we aimed to assess their satisfaction with their experience with CareTrack application.

\section{Methods}

\section{CareTrack application development}

Our group collaborated with an Information Technology firm located in London, Ontario, Canada to develop the CareTrack application. We reviewed and incorporated medical oncologists' suggestions and feedback regarding the content of this application. Prior to study start, this study was formally presentation at the thoracic multi-disciplinary tumour board at the London Regional Cancer Program and final changes based on the feedback of this presentation were added to the application. The content was felt to convey all the necessary information about the stage, extent and molecular markers of the lung cancer for patients to understand their diagnosis and treatment plan. In addition, the application had all the relevant details of the potential therapies including chemotherapy, radiation therapy and surgery. Finally, a visual diagram was also included in the application which enabled physicians to draw on an anatomical representation of the human body and lungs to better show the lung primary and metastases, if present.

\section{Study methodology}

Research ethics board approval was obtained from the University of Western Ontario Health Sciences Research Ethics Board in London, Ontario, Canada (REB\#: 111760). Initially, to recruit participants, the study was presented to eligible patients with lung cancer at their initial medical oncology consultation visit at the London Regional Cancer Program in London, Ontario, Canada. Patient recruitment began on June 20, 2018. All consented patients were then provided with a summary of the pilot study and a briefing on the extent of their participation in this qualitative survey. An iPad pre-loaded with the CareTrack application was provided to the individual oncologist at the end of the consultation, wherein they filled in the form for the respective patient. The form was filled out either in the room with the patient or by the oncologist in their office, as per the physician's discretion. A stopwatch was used to measure the time spent filling out the form by the oncologist.

After the CareTrack application form was completed, a hard printout copy was provided to the patient to take home and an e-mail was sent out with the same form as well (if the patient opted to additionally have an electronic copy). No patient identifiers were included on the printout copy or in the email. Approximately 1-2 weeks later, a patient satisfaction survey was administered over the phone and any score of $5 / 7$ or greater (questions $1,2,6,7$ ) or $2 / 7$ or greater (questions $3-5$ ) was considered meaningful/beneficial.

After completion of patient recruitment on October 31, 2019, each oncologist filled out a questionnaire to evaluate their experience with the CareTrack application (Figure 1).

\section{Results}

This study consisted of 5 oncologists who participated in the study; the average time it took for the oncologists to fill out the CareTrack application was 1 minute and 23 seconds. The results collected from the satisfaction questionnaires completed by the 5 participating physicians are listed in table 1 . Overall, physicians were comfortable documenting the consultation information and felt that the tool enhanced patient education. The majority of physicians (4/5) found the tool easy-to use and did not find it time-consuming in their clinics.

The patient satisfaction results collected from the study of 37 participants who had completed the survey, did describe an overwhelming positive effect. The survey questions with meaningful results are listed in table 2 .

\section{Discussion}

It is important for patients to understand their cancer diagnosis as well as their treatment plan, as this understanding has been found to increase patient autonomy and improve outcomes. However, in most clinical consultations, retaining information from the appointment relies solely on the memory of the patients and their loved ones to recall the discussions held by their oncologist.

With the CareTrack application, a material reference piece of information is generated for the patient that is complete with the most vital information regarding their diagnosis, stage, molecular characteristics and the treatment plan. In this study, patients found 


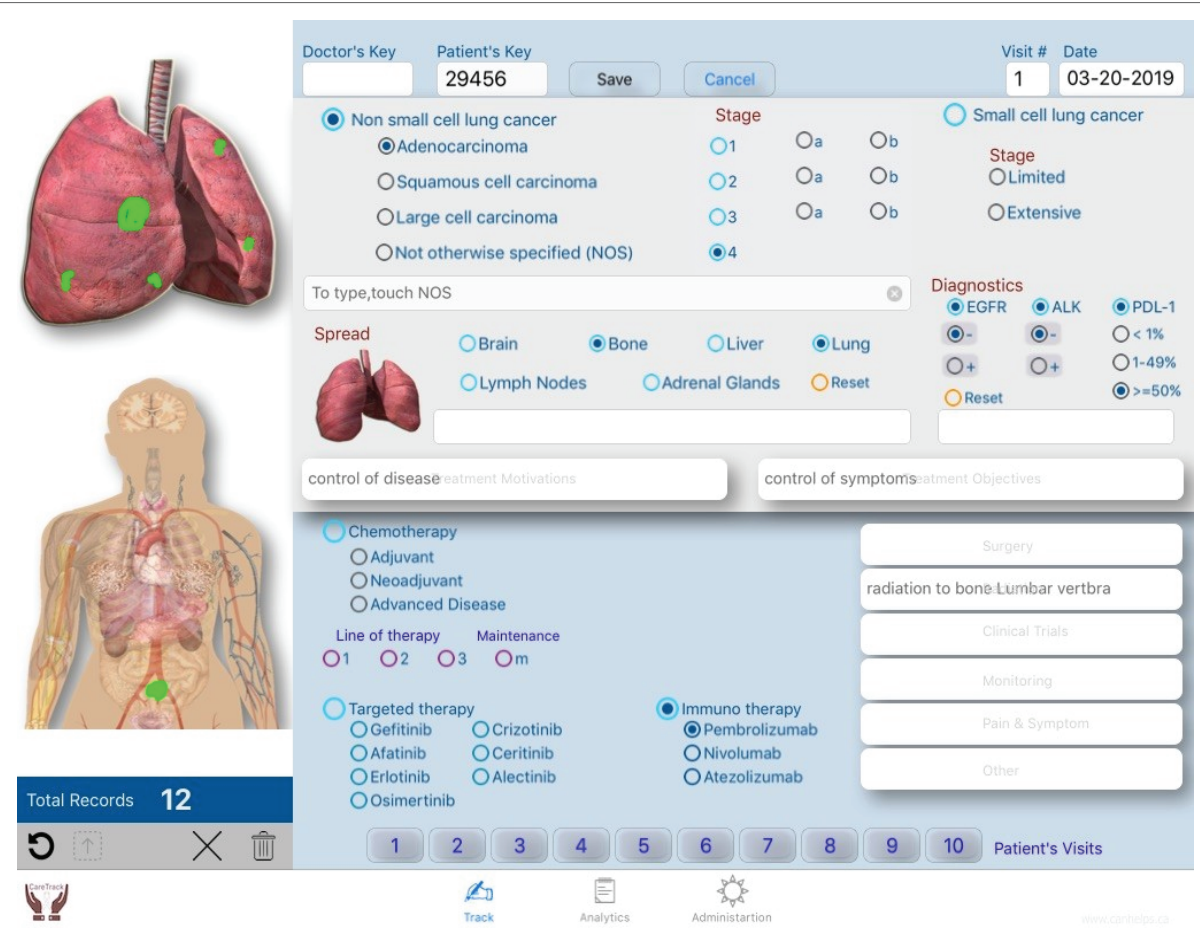

Figure 1: An example of a completed CareTrack application sheet after an initial medical oncology consultation.

Table 1: Physician satisfaction questionnaire responses.

\begin{tabular}{|l|c|}
\hline \multicolumn{1}{|c|}{ Survey questions } & $\begin{array}{c}\text { Number of physicians with responses } \\
\text { considered meaningful/beneficial }\end{array}$ \\
\hline $\begin{array}{l}\text { Did you feel that using the CareTrack tool enhanced the quality of the patient education or } \\
\text { counselling you did during the clinic visits? }\end{array}$ & $4 / 5(80 \%)$ \\
\hline How time-consuming was using the CareTrack tool? & $4 / 5(80 \%)$ \\
\hline $\begin{array}{l}\text { How comfortable were you with documenting stage and treatment intent and treatment goals for } \\
\text { the patient to read and review? }\end{array}$ & $5 / 5(100 \%)$ \\
\hline $\begin{array}{l}\text { Did you feel that the CareTrack tool was able to capture all the aspects of the treatment plan/next } \\
\text { steps? }\end{array}$ & $3 / 5(60 \%)$ \\
\hline How easy was it to use the CareTrack tool? & $4 / 5(80 \%)$ \\
\hline
\end{tabular}

Table 2: Patient satisfaction questionnaire responses.

\begin{tabular}{|l|c|}
\hline \multicolumn{1}{|c|}{ Survey questions } & $\begin{array}{c}\text { Number of patients with responses considered } \\
\text { meaningful/beneficial }\end{array}$ \\
\hline Did you find the CareTrack information provided useful? & $36 / 37(97 \%)$ \\
\hline Was the information easy to understand? & $37 / 37(100 \%)$ \\
\hline How often have you referred to the CareTrack information since your appointment? & $33 / 36(92 \%)$ \\
\hline $\begin{array}{l}\text { Has the CareTrack information helped you remember information from your } \\
\text { appointment? }\end{array}$ & $34 / 37(92 \%)$ \\
\hline $\begin{array}{l}\text { Was the CareTrack information provided referred to when your diagnosis/stage/ } \\
\text { treatment was being discussed at home? }\end{array}$ & $33 / 37(89 \%)$ \\
\hline $\begin{array}{l}\text { How comfortable are you with seeing your cancer information and treatment plan } \\
\text { displayed on the CareTrack tool? }\end{array}$ & $35 / 37(95 \%)$ \\
\hline $\begin{array}{l}\text { Would you like to receive additional CareTrack information in the future if you're staging } \\
\text { and/or treatment planned is changed/ updated? }\end{array}$ & $32 / 37(86 \%)$ \\
\hline
\end{tabular}

Citation: Vineet S, Khan F, Mirza I, Black M, Raphael J, et al. (2020) CareTrack: an Application-Based Method of Documentation for Improving 
the CareTrack application summary of their initial medical oncology consultation to be very useful and easy to understand. Patients were enthusiastic to receive this appointment summary and were comfortable viewing their cancer diagnosis and treatment plan. Additionally, most patients indicated that they would like to receive additional CareTrack information in the future, in the event that an aspect of their care plan needed to be updated and/or changed. Patients in this study were found to refer to the CareTrack summary sheet at least more than once for information and/or discussions in the home. Patients found the CareTrack sheet to be very useful when they did choose to refer to it.

With the introduction of additional tools and/or forms to fill out in a consultation, it is important that such innovations are quick and easy for physicians and/or healthcare team members to utilize in the busy oncology clinic setting. The average time to fill in a completed CareTrack application form by the oncologists was less than 1.5 minutes, which was found to be very reasonable by the participating oncologists. This additional time to their clinic was accepted as the patients found the review of their consultation very informative. This short time interval of use for the CareTrack application demonstrates that that this can be implemented for not only oncologists but for multiple different healthcare providers in everyday clinical practice. Overall, the results of this study have provided us with qualitative assurance that providing patients with a consultation summary in a printout and/or e-mail format is feasible, helpful and adoptable in the busy oncology clinic practice. To our knowledge, this is the first study of its kind that introduced a user-friendly and easy-to use application that is completed by the physician to give patients a summary of their disease and treatment. Although this was a pilot study assessing feasibility, the initial results demonstrate that the CareTrack application and summary sheet is well received by both physicians and patients.

The limitations of this study include a small number of patients and physicians sample size. In fact, 60 patients consented to participation in the study, however, only 37 patients $(62 \%)$ completed the study (i.e., were available and able to complete the follow-up satisfaction survey). The non-participation could be explained by a variety of reasons including: patients not answering their telephones when the study team attempted to reach them, patients not feeling up to participating in a survey despite initially agreeing, and patients being unexpectedly moved to palliative and/or deceased when the study team attempted to follow-up. Despite these limitations to completion of the patient satisfaction surveys, we found that all patients were initially receptive to receiving this consultation summary. In regards to the low number of physician participants, this was unfortunately a consequence of the size of our cancer centre, however, it should be noted that all possible treating lung oncologists were recruited and participated in the study. Despite the small physician sample size, the CareTrack application was found to easily integrate into the busy clinics of all participating oncologists.

This study was centred on the initial consultation visit for patients with a new diagnosis oflung cancer. Although the CareTrack application is capable of recording follow-ups and generating previously stored data, it was not utilized for this study and therefore no data is available about the follow up component. It is important to note that this was a pilot study to assess the feasibility of the CareTrack application in an oncology clinic by assessing the time it takes for physicians to complete the form. A larger study containing a comparison to a control group of patients is required in order to conclusively assess the benefit of the CareTrack application on patient communication of clinical oncology information.

\section{Conclusions}

In the future, further research on the kind of information that patients actually want to know and how to make it more accessible to them would be valuable. Additionally, a better method to communicate with patients for the follow up survey would improve data collection and breadth. Future steps should include aiding hospitals in embedding a system like the CareTrack application in all oncology fields especially, as well as further implementing this technology in other field such as cardiac, thoracic, etc.

\section{Acknowledgments}

We would like to acknowledge Tariq Khan (Canris Technologies) for providing the iPad and printer as well as for his assistance in developing the CareTrack iPad application software. We would also like to thank the Division of Medical Oncology at the London Regional Cancer Program for their support and participation in this study.

\section{References}

1. Cassileth BR, Zupkis RV, Sutton-Smith K, March V (1980) Information and participation preferences among cancer patients. Ann Intern Med 92: 832-836.

2. Harrison-Woermke DE, Graydon JE (1993) Perceived informational needs of breast cancer patients receiving radiation therapy after excisional biopsy and axillary node dissection. Cancer Nurs 16: 449455.

3. Kessels RP (2003) Patients' memory for medical information. J R Soc Med 96: 219-222.

4. Mossman J, Boudioni M, Slevin ML (1999) Cancer information: a cost-effective intervention. Eur J Cancer 35: 1587-1591.

5. Tran C, Dicker AP, Jim HSL (2017) The Emerging Role of Mobile Health in Oncology. J Target Ther Cancer.

6. Krist AH, Woolf SH, Hochheimer C, Sabo RT, Kashiri P, et al. (2017) Harnessing Information Technology to Inform Patients Facing Routine Decisions: Cancer Screening as a Test Case. Ann Fam Med 15: 217-224.

7. Raghunathan NJ, Korenstein D, Li QS, Tonorezos ES, Mao JJ (2018) Determinants of mobile technology use and smartphone application interest in cancer patients. Cancer Med 7: 5812-5819.

8. Nasi G, Cucciniello M, Guerrazzi C (2015) The role of mobile technologies in health care processes: the case of cancer supportive care. J Med Internet Res 17: e26.

9. Gonzalez BD (2018) Promise of Mobile Health Technology to Reduce Disparities in Patients with Cancer and Survivors. JCO Clin Can Inform 2: 1-9. 